\title{
BMJ Open Lessons from countries implementing find, test, trace, isolation and support policies in the rapid response of the COVID-19 pandemic: a systematic review
}

\author{
Sheng-Chia Chung (D) , ${ }^{1}$ Sushila Marlow, ${ }^{2}$ Nicholas Tobias, ${ }^{3}$ Alessio Alogna, ${ }^{4}$ \\ Ivano Alogna, ${ }^{5}$ San-Lin You, ${ }^{6,7}$ Kamlesh Khunti, ${ }^{8}$ Martin McKee (i) , ${ }^{9}$ \\ Susan Michie, ${ }^{10}$ Deenan Pillay ${ }^{11}$
}

To cite: Chung S-C, Marlow S, Tobias N, et al. Lessons from countries implementing find, test, trace, isolation and support policies in the rapid response of the COVID-19 pandemic: a systematic review. BMJ Open 2021;11:e047832. doi:10.1136/ bmjopen-2020-047832

- Prepublication history and additional supplemental material for this paper are available online. To view these files, please visit the journal online (http://dx.doi.org/10.1136/ bmjopen-2020-047832)

Received 10 December 2020 Revised 19 April 2021 Accepted 21 April 2021
Check for updates

(C) Author(s) (or their employer(s)) 2021. Re-use permitted under CC BY-NC. No commercial re-use. See rights and permissions. Published by BMJ.

For numbered affiliations see end of article.

Correspondence to Dr Sheng-Chia Chung; s.chung@ucl.ac.uk

\section{ABSTRACT}

Objective To systematically learn lessons from the experiences of countries implementing find, test, trace, isolate, support (FTTIS) in the first wave of the COVID-19 pandemic

Design, data sources and eligibility criteria We searched MEDLINE (PubMed), Cochrane Library, SCOPUS and JSTOR, initially between 31 May 2019 and 21 January 2021. Research articles and reviews on the use of contact tracing, testing, self-isolation and quarantine for COVID-19 management were included in the review.

Data extraction and synthesis We extracted information including study objective, design, methods, main findings and implications. These were tabulated and a narrative synthesis was undertaken given the diverse research designs, methods and implications.

Results We identified and included 118 eligible studies. We identified the core elements of an effective find, test, trace, isolate, support (FTTIS) system needed to interrupt the spread of a novel infectious disease, where treatment or vaccination was not yet available, as pertained in the initial stages of the COVID-19 pandemic. We report methods used to shorten case finding time, improve accuracy and efficiency of tests, coordinate stakeholders and actors involved in an FTTIS system, support individuals isolating and make appropriate use of digital tools.

Conclusions We identified in our systematic review the key components of an FTTIS system. These include border controls, restricted entry, inbound traveller quarantine and comprehensive case finding; repeated testing to minimise false diagnoses and pooled testing in resource-limited circumstances; extended quarantine period and the use of digital tools for contact tracing and self-isolation. Support for mental or physical health and livelihoods is needed for individuals undergoing self-isolation/quarantine. An integrated system with rolling-wave planning can best use effective FTTIS tools to respond to the fast-changing COVID-19 pandemic. Results of the review may inform countries considering implementing these measures.

\section{Strengths and limitations of this study}

- The study systematically reviews the core elements of an effective find, test, trace, isolate, support (FTTIS) system to interrupt the spread of COVID-19. Our findings can inform policy in future pandemics.

- We reported optimal strategies reported in the literature to shorten case finding time, improve both accuracy and efficiency of tests, coordinate stakeholders and actors involved in an FTTIS system, support individuals as they progress from case finding to isolation and make appropriate use of digital tools to facilitate the programme.

- The main limitation is the scarcity of quantitative studies.

\section{INTRODUCTION}

Interrupting transmission of COVID-19 has depended on rapid isolation of infected individuals. For this to happen, a complex set of co-ordinated elements must be implemented to find potential cases, either by identifying those who have symptoms or examination of individuals at risk, test to confirm the presence of infection, trace contacts, isolate those infected and their contacts ${ }^{1}$ and support ${ }^{2}$ those in isolation to reduce the risk that they will breach any restrictions.

While various elements of a find, test, trace, isolate, support (FTTIS) system have long been core elements of the public health role, COVID-19 infection has some specific characteristics, such as transmission by presymptomatic and asymptomatic individuals ${ }^{3}$ and a strong age gradient in disease severity as well as other features that remain poorly understood. Despite this uncertainty, some countries have implemented successful COVID-19 FTTIS programmes, which have achieved 
coordination among relevant actors, including central and local government authorities, laboratories and the medical care system. ${ }^{1}$ These actors are interconnected by integrated real-time information flow. However, the ways that they have created these systems have varied, largely determined by existing structures.

There are no simple solutions. Thus, digital tools were initially seen as making a major contribution to FTTIS programmes. ${ }^{13}$ Yet while the English National Health Service (NHS) contact tracing app showed positive results in a pilot study, decreasing rates of transmission, ${ }^{4}$ it struggled to achieve a sufficient level of uptake to be effective. ${ }^{5}$ Individuals, organisations or occupational groups with high COVID-19 risk need additional support to use such apps $^{2}$ while it can be challenging to reduce the burden of unnecessary or repeated episodes of quarantine.

The main challenges in implementing an FTTIS programme include developing a strategy that can reduce delays in finding cases and contacts, ${ }^{1}$ ensuring the accuracy of tests, ${ }^{1}$ establishing integrated systems ${ }^{67}$ reducing barriers to adhering to regulations, especially isolating ${ }^{89}$ and mitigating adverse economic impacts on the livelihood of individuals affected. ${ }^{9}$ To inform and support the design, implementation and continuous improvement of an FTTIS system, we conducted a systematic review, structured to answer a series of key questions that arose during the initial response to the COVID-19 pandemic. These policy questions addressed the following issues: (1) infrastructure and processes needed to achieve a samplingto-results process taking 24 hours or less, (2) adherence to isolation and local support needs, (3) real-time data management, linkage of datasets and dashboards and ownership of the data generated, (4) achieving a 'rapid response', (5) assimilation of an application (app) in light of the above, (6) overcoming the barriers to and enablers of being tested, reporting contacts and isolating as a result of being contacted.

\section{METHODS}

The systematic review follows the Preferred Reporting Items for Systematic Reviews and Meta-Analyses guideline. The review protocol is described as the following.

\section{Eligible studies}

Eligible studies are research articles and reviews evaluating the strategies of contact tracing, testing, self-isolation and quarantine on COVID-19 management published in English worldwide during the urgent response of the COVID-19 pandemic (May 2019 to May 2020 and June 2020 and January 2021).

\section{Information sources and search}

We searched MEDLINE (PubMed), Cochrane Library, SCOPUS and JSTOR. The search terms included 'contact tracing' or 'testing' or 'self-isolation' or 'quarantine' in the title in combination with 'COVID-19' or 'COVID19 ' or 'coronavirus' in the title or text. The full-search strategy is included in the online supplemental appendix 1 section 1 , and we apply the same search criteria for all the literature databases. The initial search included material published up to 28 May 2020. Studies were limited to those in which the subjects were human. Relevant reports and literature cited in the papers identified as well as material from other sources such as the COVID-19 Response Monitor developed by the European Observatory on Health Systems and Policies were also considered. ${ }^{10}$ The initial search was subsequently updated to include material published between June 2020 and January 2021 (online supplemental appendix 1 section 2).

\section{Study selection}

In the MEDLINE search, clinical studies, clinical trials, evaluation studies, government documents, journal articles, multicentre studies, observational studies, practice guidelines, pragmatic clinical trials and technical reports were included, as were reviewed. Studies whose subject areas were in medicine or social sciences in SCOPUS, and research reports in JSTOR (excluding book chapters) were selected. We screened title, abstract and content to remove studies unrelated to elements of FTTIS or COVID-19.

\section{Data collection process and data items}

For each publication, we extracted (where appropriate) information on study design, method, results, main findings and limitations using the PICOS tool. ${ }^{11}$

\section{Summary measures and synthesis of results}

Following discussion with researchers involved in the COVID-19 response in the UK (https://www.independentsage.org/), a list of key questions to answer was developed and answered using the literature collected. Sheng-Chia Chung, Sushila Marlow and Nicholas Tobias screened studies and extracted the data and disagreements were resolved through discussions.

\section{Risk of bias in individual studies}

As studies eligible for policy review were mostly qualitative, we managed by using the Critical Appraisal Skills Programme (CASP) tool to assess the methodological rigour of included studies ${ }^{12}$ (CASP Qualitative Studies Checklist). ${ }^{13}$

\section{Patient and public involvement}

The study questions were inspired by weekly public discussions held by Independent SAGE, a scientific group for COVID-19 response in the UK (https:/ / www.independentsage.org/).

\section{RESULTS}

In the initial search, we identified 50 studies from PUBMED (Medline), 43 studies from SCOPUS, 56 from JSTOR, 1 from the Cochrane library and four from additional sources. The flow diagram is set out in figure 1. From the 164 initial publications, we removed 


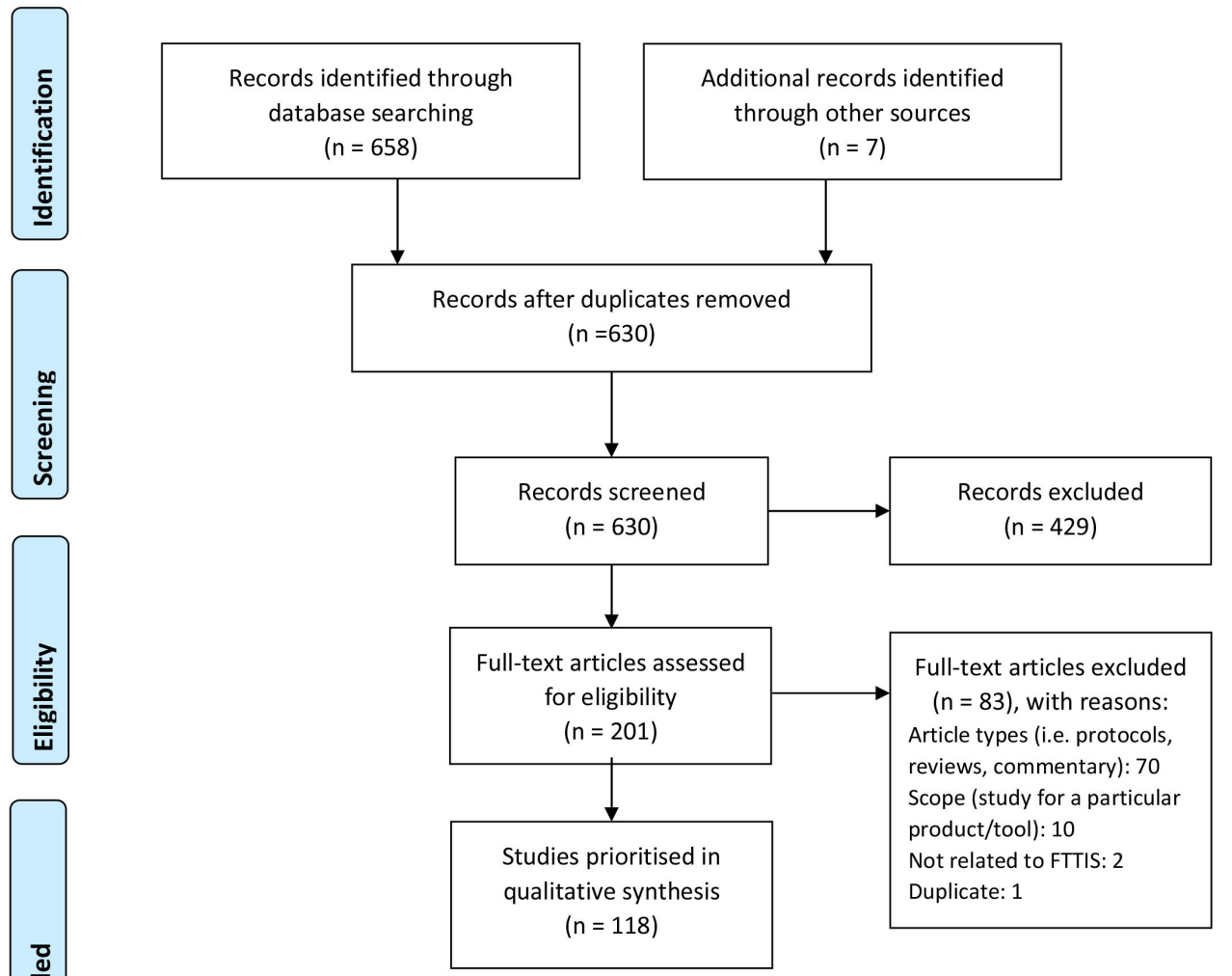

Figure 1 Systematic review study flow diagram. FTTIS, find, test, trace, isolate, support.

30 duplicate studies, 84 items were not directly focused on COVID-19 or strategy of testing, contact tracing or isolation or not in the format of a research report (such as editorials or podcasts). We subsequently identified an additional 58 studies in the second supplementary systematic review discussing FTTIS strategies during the early phase of the COVID-19 pandemic published between June 2020 and January 2021. We, thus, included a total of 118 studies in the review (online supplemental table S1). Eligible studies had clearly stated aims and applied appropriate methods, and although none addressed all proposed study questions, they provided data relevant to an aspect in our review (online supplemental table S2). A full list of studies included in the review is listed in online supplemental appendix 1 section 3.

\section{Overview of FTTIS}

We initially review some key findings on the COVID-19 pandemic. A review by Nussbaumer-Streit B et al concluded that a combination of quarantine and other nonpharmaceutical interventions could significantly reduce COVID-19 incidence and mortality. ${ }^{14}$ Policy decisions should be informed by a detailed understanding of transmission dynamics. With growing evidence that so-called super-spreading events played an important role in many outbreaks, ${ }^{15}$ finding, testing and isolating those present at such events was recognised as a priority. ${ }^{16}$ This relates to work by Manchein et al who proposed that the growth of COVID-19 cases follows a power-law pattern rather than an exponential one, suggesting that transmission is characterised by features of scale-free networks, fractal kinetics and small world features. The implication is that while social distancing has been important to slow the spread of COVID-19 so far, a robust testing and isolation would be necessary to facilitate economies to reopen. ${ }^{17}$ These conclusions were consistent with work from Italy stressing the importance of maintaining very strict quarantine of those affected. ${ }^{18}$ Policies must also be underpinned by high-quality population-level data, yet some countries have struggled to create well-functioning integrated systems. Inadequate testing will often underestimate the true number and growth rate of COVID-19 cases. ${ }^{190}$ This is a challenge because testing is at the heart of a comprehensive FTTIS system and the only way to make a diagnosis where symptoms are atypical or unclear or in cases that are asymptomatic or presymptomatic. ${ }^{21}$ For incidence, analysing surveillance data found that the high case fatality during the first 40-day COVID-19 epidemic in Italy was primarily due to insufficient testing to characterise the outbreak accurately, thus masking transmission in the community and healthcare facilities. ${ }^{22}$ In countries where FTTI interventions have been implemented subsequently, there has sometimes been a delay in their effect on disease control. ${ }^{23}$ There are now a number of reports of how FTTIS systems have been designed and operated. ${ }^{24}$ 
Infrastructure and procedure of a sampling-to-results process that is $\mathbf{2 4}$ hours or less

Countries have differed in whether they have built on existing infrastructure or created new structures to deal with the pandemic. We have identified two complementary elements in the reviewed studies, one involves increasing sampling capacity and the other scaling up laboratory capacity. The UK addressed the first of these by implementing drive-through SARS-CoV-2 testing ${ }^{25}$ with patients being informed of their results within 24-36 hours. It created a separate network of new standalone laboratories. Lagier et al reported on SARS-CoV-2 reverse transcription-PCR (RT-PCR) testing of repatriated French citizens during their initial response, ${ }^{26}$ with time from sample to result reduced to 3 hours by prioritising extraction of RNA from samples. Replacing the RNA extraction step by performing RT-PCR directly on heatinactivated or lysed samples could accelerate the process further. ${ }^{27}$ Point of care testing could also reduce the time to results to less than 2 hours. ${ }^{28}$

Binniker has set out the steps that should be taken when scaling up activity in existing laboratories, ${ }^{29}$ which has the advantage of using networks already in place, including transport and communications. Similarly, Gupta et al have described how laboratories in universities and clinics in India were converted into testing centres. ${ }^{30}$ In Ethiopia, Abera et al reported using Malaria and Neglected Tropical Diseases laboratories for COVID-19 testing. ${ }^{31}$ Tolia $e t$ al described the implementation of a testing programme within a hospital emergency department. ${ }^{32}$

\section{Strategies to improve the testing response}

The case definition of potential COVID-19 changed frequently in the initial stage of the epidemic, as knowledge of the disease evolved. In the early stage of the epidemic, test kits and laboratory capacity were in short supply in many countries. ${ }^{33}$ An example of an early investigation is when the Dutch National Outbreak Management Team investigated a potential SARS-CoV-2 local transmission, with rapid testing of healthcare workers in an area with a suspected COVID-19 outbreak. Testing followed a national protocol and was carried out either locally or in central laboratories. Nine hospitals were asked to sample healthcare workers on Saturday, 7 March and results were due on Monday, 9 March. When the SARS-CoV-2 positive rate came back as 4.1\% among 1097 tested, the local outbreak was confirmed.

Other laboratory-based strategies to improve testing efficiency include Yan et al suggesting placing samples in reagents containing guanidine salts, for example, TRIzol, TRIzol LS or AVL buffer, to inactivate the virus and protect RNA. ${ }^{34}$ Pooled testing approaches have been advocated to increase testing capacity ${ }^{35} 36$ and facilitate the detection of asymptomatic cases. ${ }^{37}$ Only if the pooled batch tested positive would sample contributing to the batch be tested individually. ${ }^{38} \mathrm{~A}$ three-stage pooling system was reported by Eberhardt $e t$ al who showed that it was possible to test three and seven times as many individuals with the same number of test kits when prevalence rates were $5 \%$ and $1 \%$, respectively. ${ }^{39}$

Several studies examined strategies to test high-risk individuals, ${ }^{40}{ }^{41}$ concluding that it had potential if well targeted,${ }^{42}$ whereas the utility of asymptomatic testing in low-prevalence setting may be low. ${ }^{43}$ Targeted testing, measured by tests per confirmed case, was reported to be correlated with a greater reduction in COVID-19 fatality than population testing number (tests per million people) in The Organisation for Economic Co-operation and Development (OECD) countries and Taiwan. ${ }^{44}$ One measure of the performance of a contact tracing and testing system is a shorter interval between the diagnosis of the index and secondary cases. ${ }^{45} 46$

\section{Minimising false test results}

Inaccurate results can impede both the treatment of the patient and public health interventions to contain COVID-19 infection. ${ }^{47}$ Testing accuracy may be influenced by sampling and testing kit performance. The gold standard for COVID-19 diagnosis remains RT-PCR-based assays with respiratory specimens, although these can give positive results due to the persistence of fragments of genetic material after the individual has ceased to be infectious. ${ }^{48}$ Among different respiratory sampling methods, RT-PCR assays based on nasopharyngeal swabs tend to have a lower cycle threshold than oropharyngeal swabs. ${ }^{34}$ Infected individuals who are initially RT-PCR negative are likely to become positive with repeated tests as the infection progresses, ${ }^{4950}$ reflecting differences in the amount of virus at the site samples and viral shedding at the time of sampling. ${ }^{49-51}$ Sampling error can occur due to low levels of virus at the beginning or end period of shedding or inadequate sampling techniques. Thus, recommendations are to take multiple samples, ${ }^{49} 5152$ while some have proposed supporting RT-PCR results with Chest CT imaging. 4853

The performance of tests has improved over time. Thus, low sensitivity (30\%) was reported in the RT-PCR testing kits in the early stage of the pandemic. ${ }^{54}$ In a review by Younes et al, RT-PCR testing kits developed in the USA, France and Germany had a high sensitivity, at $95 \%$, but specificity was not reported. ${ }^{48}$ Yan et al have suggested that human RNase P gene could be amplified as an internal control to reduce false-negative results and template volume could be increased to improve sensitivity. ${ }^{34}$ One study from March 2020 in India found no false negatives but only five in seven positive tests were confirmed. ${ }^{30}$ As a consequence, all apparent positive tests were retested by the National Institute of Virology. Point of care rapid testing has obvious benefits in terms of speed of results ${ }^{55}$ but Döhla et al reported that tests available in the initial period of the pandemic had low sensitivity (36.4\%) compared with RT-PCR, although this was in a small group of 39 patients with COVID-19. ${ }^{51}$

Besides RT-PCR testing, serologic antibody assays detect immunoglobin $\mathrm{G}$ and immunoglobin M to SARS-CoV-2 after infection. ${ }^{56}$ This has been used to determine 
whether an individual or the proportion of a population has previously been infected. ${ }^{57}$ Given concerns about the duration of antibodies and limitations in sampling and testing, there is a need for caution about potential falsenegative results, and repeated testing may be required if symptoms persist. Testing at the end of the quarantine may help identify and care for delayed or asymptomatic COVID-19 cases while preventing further transmission. ${ }^{58}$

In addition to the aforementioned challenge relating to false-negative results, concern has been raised about the specificity of rapid antigen assays, with the risk of a high proportion of false positives, particularly as overall population prevalence reduces. For this reason, some countries are instigating PCR confirmation of positive rapid test results. ${ }^{59}$

\section{Linked data to facilitate active case finding}

There are several examples of how linked health service data can be used to identify cases. ${ }^{24} 60$ For example, the digital COVID-19 border control system in Taiwan collects the health information of inbound passengers. The data are linked to the 'epidemic prevention tracking system' used by health authorities to ensure isolation and quarantine of cases and are linked to the National Health Insurance MediCloud System, a cloud-based platform for medical professionals to gain updated clinical data of the patient seeking care. ${ }^{61}$ The data linkage provides medical staff with real-time information on the patient's COVID-19 travel and contact history, home isolation and quarantine status, to provide necessary care with appropriate infectious control measures ${ }^{62}$ Screening high-risk populations (such as healthcare workers) has been used for active case finding. ${ }^{63}$

\section{Adherence to isolation and local support needs \\ Inbound traveller quarantine}

Border controls and restricted entry were imposed in East Asian countries early in the pandemic,${ }^{54}$ linked to comprehensive inbound traveller quarantine. For example, Singapore imposed a 14-day 'Stay Home Notice' (SHN) on visitors and returning residents from COVID-19 endemic areas since January 2020 and for all inbound traveller from 9 April 2020. On arrival, travellers underwent a 14-day mandatory stay in government-designated hotels. During this period, they were not allowed to leave their individual rooms, where specific infectious disease prevention procedures were implemented. ${ }^{64}$

\section{Timely identification of contacts}

Ferretti et al reported an estimated reproduction number of the initial SARS-CoV-2 variants of 2.0, of which 0.9 was associated with infection occurring during the presymptomatic stage, suggesting about half of the cases were infected by patients with COVID-19 before symptom onset. ${ }^{65}$ An individual infected by SARS-CoV-2 can actively shed the virus from their respiratory system 6 days before and 3 weeks after displaying symptoms, depending on disease severity. ${ }^{3448}$ While the virus detected in respiratory samples peaked within the first week of the illness, virus shedding continues in stool samples and peaks in week 2 or $3 .^{34}$ It, thus, is essential to stop the transmission by rapidly finding potential cases and their contacts and quarantining. ${ }^{66}$ One modelling study reported how minimising testing delay effectively reduced onward transmission. ${ }^{67}$ Countries may apply an additional week of self-health management beyond the 2-week quarantine period. ${ }^{24}$

Modelling studies have indicated that strategies to reduce random community contacts are preferable to those based solely on reducing personal contacts, ${ }^{40} 68$ the duration of quarantine can vary depending on the reduction of contacts reached by individuals ${ }^{68}$ and testing at the beginning and the end of quarantine. ${ }^{69}$ The definition of contact may vary; Korea applied a focused testing strategy where, when a COVID-19 outbreak with an unknown source of infection occurred, there was the testing of individuals who were working, living or visiting in the outbreak area. ${ }^{70}$ Individuals who tested positive were hospitalised, whereas individuals who tested negative were self-quarantined for 14 days. The strategy effectively reduced the proportion of COVID-19 cases with an unknown source of infection and the risk of transmission in the community. ${ }^{70}$ The direction of contact tracing may be forward or both forward and backward; the latter identifies unascertained or asymptomatic carriers and enables the isolation of those involved in otherwise undiscovered transmission. ${ }^{28}$

\section{Digital tools to facilitate FTTIS}

Conventional epidemiological contact tracing, which relies on personal interviews, is labour intensive and timeconsuming and may be challenging during a large-scale epidemic. ${ }^{241}$ Some countries have applied digital tools in COVID-19 responses. Measures that use automated monitoring of geolocation data generated automatically by smartphones have advantages over voluntary tools (such as apps), but there may be differing concerns about privacy among countries. ${ }^{72-74}$

Types of telecommunication provider-based measures may include:

1. Mapping anonymised cell phone movement in a particular area (eg, Germany, Austria, Italy).

2. Base station triangulation to approximate cell phone location (Taiwan and Hungary) ${ }^{75}$ or

3. Access to Global Positioning System (GPS) data generated by the phone (Israel).

Voluntary provision of data includes:

1. An app that captures proximity to other enabled mobile devices within a specified range via Bluetooth (Singapore, Austria).

2. An App recording daily symptoms (South Korea, Taiwan, Poland, UK) or

3. A Quick Response (QR) code for entry or exit to key locations (China).

Aslam et al reviewed the digital tools used in East Asian countries for COVID-19 response by their level of 
intrusiveness. ${ }^{54}$ Low intrusive digital tools provide services to individuals and act as the interface between authorities and service users. ${ }^{60}$ Such tools are generally widely accepted. Other tools are used to enforce quarantine and may involve individual movement restrictions and privacy concerns ${ }^{76}$ These are subjected to debate and have lower acceptability, yet large-scale uptake $(60 \%-75 \%$ of the population) may be necessary for them to be effective. ${ }^{73}$ For example, in Singapore, the initial voluntary use of the contact tracing app was only $12 \%{ }^{73}$ The use of such apps requires a clear legal basis for the information that can be used exclusively for infectious disease control and comes with strong legal limits on data access. ${ }^{54} 74$

\section{Support for individuals complying with self-isolation}

Webster et al identified factors associated with adherence, which included the knowledge people had about the disease and quarantine procedures, social norms, perceived benefits of quarantine and perceived risk of the disease as well as practical issues such as access to essential supplies or the financial consequences of being out of work. ${ }^{77}$ Self-isolation or quarantine may not be affordable for low wage and informal workers. ${ }^{78}$ To address financial needs, the Singaporean government provides self-employed persons and businesses with employees undergoing self-isolation or quarantine (SHN) a US $\$ 100$ per day support. Deliveries of food and other supplies can be arranged for individuals in SHN, through a designated hotline. By law, landlords and dormitory operators cannot evict tenants in SHN. For individuals in residences may not be suitable for SHN, the government offers hotels as an alternative. ${ }^{64}$

Psychological stress, ${ }^{77} 7980$ cognitive dissonance ${ }^{74}$ or lack of physical activities ${ }^{81}$ during self-isolation may adversely affect mental or physical health. While anxiety was reported to be positively associated with an interest in security-related actions, perceived restriction to personal freedom had a negative impact on taking these actions and loneliness related to nonaction. ${ }^{82}$ In a rapid review, Brooks et al reported individuals in quarantine experiencing confusion, anger and post-traumatic stress symptoms, for reasons such as fear of infection, frustration, boredom and inadequate supplies. ${ }^{77}$ Austria and Germany reported an increase in domestic violence associated with COVID-19 restrictions. ${ }^{83}$ Strategies to mitigate these adverse effects include timely and sufficient information to reduce uncertainty, minimising the quarantine period to no longer than is necessary and providing adequate supplies for individuals serving self-isolation.

Razai et al suggested using validated psychological screening tools, such as the UCLA Loneliness Scale and Patient Health Questionnaire-4 for anxiety and depression to identify individuals who need support. This can help identify those who may benefit from counselling, coaching via telephone or online video consultations. ${ }^{80}$ Nonmedical social prescribing, such as visual choirs, online theatre or performances, exercise or art classes can reduce boredom. Communication using social media may ease loneliness. Banskota et al reviewed smartphone apps to assist older adults in coping with self-isolation, designed for social networking, food and drinks delivery, medical consultation, health and fitness. ${ }^{84}$ Meinert et al described an agile process to develop an app for older people and their families to improve well-being while observing social distancing rules. ${ }^{85}$

How would real-time data management, linkage of data sets and dashboards be developed, and who would 'own this'?

In Austria, the nationwide online COVID-19 dashboard has facilitated effective early response to COVID-19 pandemic. ${ }^{83}$ In South Korea, the government links digital databases to facilitate contact tracing, including electronic health records, phone-based GPS, card transaction records and closed circuit television. ${ }^{72}$ Yasaka et al have proposed the use of an app with three guides, requiring users to register with checkpoints (eg, public spaces or shops with a QR code that can be scanned to the app), check risk level and report COVID-19 status. ${ }^{86}$ Likewise, in China, a QR code-based app is used to limit the movement of suspected patients with COVID-19 and displays a green, amber or red code that is required to be in or enter locations, but the digital surveillance architecture and data protection mechanisms remain unclear. ${ }^{54} 7374$ Consequently, public concerns about data protection and privacy have been raised in respect to both the Chinese and South Korean apps.

The efficacy of mobile positioning data (although not possible for $2 \mathrm{G}$ phones) was studied in Nigeria by Ekong et al based on systems used in South Korea, Singapore and China. ${ }^{87}$ A legal framework for data protection was suggested when implementing these systems and a thirdparty agreement was put in place for data use.

\section{Mobile geopositioning data}

Mobile geopositioning has been used to study the mobility, connectivity and health risks in travellers. ${ }^{88}$ These have an accuracy of 150 metres, so they reduce the risk of undermining individual privacy. ${ }^{24}$ Taiwan has applied a geopositioning method to facilitate adherence to home isolation or quarantine, rapidly identifying contacts in a suspected large-scale outbreak. An example is the identification of 627386 contacts of the 3000 Diamond Princess passengers touring in Taiwan during a COVID-19 outbreak. ${ }^{24}$ The process took a day and text messages advising self-health management were sent to all contacts. Contact data were linked to electronic health records for follow-up and testing of symptomatic contacts. Geopositioning data have lessened the pressure on health authorities who would otherwise have relied on resource-intensive manual contact tracing, facilitating timely large-scale outbreak containment. The resources saved by leveraging technology can then be used for taking care of vulnerable populations and those without access to a mobile phone. 
How would a 'rapid response' occur and what would precipitate such a response?

On 22 January, an individual from Wuhan who had travelled to Hong Kong reported respiratory symptoms, and a second suspected case was reported the next day. Both received medical care, being placed in isolation and later testing positive for COVID-19. Contact tracing started immediately and the travel histories of patients were retrieved and published online. All their close contacts, including passengers seated close by and taxi drivers, were subjected to quarantine at a holiday village converted to a quarantine centre to host contacts of confirmed cases. A hotline was set up to answer public enquiries, especially for passengers on the same train/flight. ${ }^{85}$

In Singapore, proactive contact tracing and cluster identification are key features of the country's COVID-19 response. Health professionals are trained to identify potential outbreak clusters, asking a series of questions to patients with COVID-19. The Ministry of Health works with hotels to develop quarantine sites and liaises with those possessing the closed-circuit television footage to track cases. ${ }^{85}$

\section{Infection control}

Inadequate infection control during isolation or quarantine may increase the transmission of SARS-CoV-2. Close quarter isolation was found to be ineffective ${ }^{89}$ in the case of the Diamond Princess. The virus spread to 634 passengers despite contact tracing ${ }^{90}$ and safety measures, ${ }^{91}$ leading the authors to conclude that aerosol transmission played an important role in confined settings. However, this was questioned by Wang et al in a hospital study of transmission via air, sewage, surfaces and personal protective equipment in which swabs tested positive only from sewage. ${ }^{92}$

\section{Nosocomial infection}

Nosocomial infection and cross infection of health workers were reported at the Sheffield Teaching Hospitals NHS Foundation Trust; of 1533 symptomatic healthcare workers, 18\% were positive for SARS-CoV-2. ${ }^{93}$ It was estimated that a third of staff had completed a shift while symptomatic, emphasising the need for regular and efficient testing for healthcare workers who have a high risk of infection, for the protection of vulnerable patients and civilians. A study in Taiwan on the risks of contact infection before and after symptom onset showed that the spread of COVID-19 was highest within the first 4 days of infection. In comparison with transmission to medical staff, most transmissions took place in the family or social settings, partially due to the absence of the personal protective equipment (PPE) and partially due to contact in the early stages of infection (presymptomatic) ${ }^{94}$

To control nosocomial infection, Taiwan implemented nationwide enhanced Traffic Control Bundling (eTCB) in hospitals, whereby infection was controlled with a combination of triage prior to hospitalisation, separation between risk zones, strict PPE use and hand disinfection checkpoints. ${ }^{60} 95$ Risk zones were divided into the contamination, intermediate and, finally, clean. This drew on earlier research by Yen et al in which SARS infection among healthcare workers in the eTCB hospital was 2(0.03 cases per bed) compared with 50 probable cases ( 0.13 cases per bed) in the control group. ${ }^{96}$ This strategy was implemented across Taiwan on 21 May 2003 and within 2 weeks the epidemic was under control.

\section{How would an app be assimilated in light of the above?}

The Singaporean government has developed the App 'TraceTogether' to support manual contact tracing, ${ }^{97}$ recording other users who have been in proximity to a smartphone user via Bluetooth. After a user is found to be positive, individuals at risk are contacted directly. ${ }^{5474}$ The Pan-European Privacy-Preserving Proximity Tracing coalition proposed a privacy-friendly contact tracing apps with the use of matching Bluetooth signals, based on the Singaporean 'TraceTogether' app. ${ }^{73}$ The Korean Ministry of the Interior and Safety has developed a mobile phone application named 'self-quarantine safety protection' app that monitors the location of the quarantined user, informs health authorities, allows the user to report on their symptoms, and health officials can evaluate if a test is needed. ${ }^{72}$ TRACE, the national contact tracing programme in Taiwan, developed in 2017 has an integrated system to monitor health, trace contacts and link to other databases for a range of infectious diseases. The location of individuals could be tracked and communication between the user, Taiwan Centers for Disease Control and Prevention (CDC), police and authorities as well as local health departments could be centralised through this platform for reporting symptoms and contact tracing. ${ }^{98}$

What are the barriers to and enablers of being tested, reporting contacts and isolating as a result of being contacted?

\section{Logistics}

A lack of public health funding may weaken outbreak preparedness. ${ }^{99}$ Regionalisation of the healthcare system might result in fragmentation of the epidemic response and timely data availability. ${ }^{22}$ Scarcity of healthcare resources creates major barriers to COVID-19 control. ${ }^{100}$

\section{Testing}

With the USA in mind, Parmet et al encouraged the promotion of 'free testing' in order to reach poorer communities and eradicate the infection. ${ }^{78}$ Although the UK implemented free testing for adults, there have been challenges in communicating information on how to obtain a test and in what circumstances, particularly among certain ethnic minority groups. Understanding the factors associated with testing uptake in the population may inform better testing strategies. ${ }^{101}$ Mark et al reviewed the feasibility of a mobile community testing team in Scotland and reported a lack of guidance on infection control for testing in the community, thereby risking cross-transmission. Other barriers included the shortage of staff, ${ }^{102}$ shortage of testing kits and strict testing eligibility, which excludes asymptomatic patients from receiving testing on time. ${ }^{83}$ 


\section{Contact tracing}

Validity and reliability of information recorded by an app may not be accurate or precise due to technological limitations. ${ }^{84}$ Klonowska et alsummarised the high coverage needed for an app to be effective. This may be difficult to reach on a voluntary basis, for issues such as mobile storage data, operating systems and battery power to support constant Bluetooth activation, the fact that young children and senior citizens may not carry or own a personal smart device and the issue of individual willingness. ${ }^{73}$ In the UK, only $47 \%$ of individuals who are 75 years or older use the internet. ${ }^{103}$ Senior citizens in the Netherlands were also less likely to adopt a contact tracing app than their younger peers. ${ }^{104}$ It is essential that outbreak control teams cater to their needs and those of community volunteers, such as the NHS volunteer responders in the $\mathrm{UK}^{80}$

\section{Isolation}

Logistic challenges arise in providing food, sanitation, transport ${ }^{105}$ and care for individuals living in a restricted zone, especially if this covers a large area. ${ }^{78}$

\section{Public awareness and communication}

The number of tests carried out during an emergency will depend on public perception of the reliability of testing services and the effectiveness of communication of actions that can be taken. It is also necessary to ensure that kits and the location of testing stations are accessible to disadvantaged groups who may not have access to a smartphone or basic supplies. Where ethnic minorities are at greater risk, special efforts must be made to encourage them to access tests. Regular press conferences by authorities, briefing on the progress of the pandemic, changes in policies and correcting misinformation $^{60}$ can increase public awareness of the need for FTTIS and tackle rumours that arise where there are knowledge gaps and uncertainties. ${ }^{95}$ However, underreporting of COVID-19 deaths by the government may occur in countries with incomplete vital registration or under political influence. $^{20106}$

The internet and social media have an important influence on behaviour in isolation, ${ }^{107}$ which has grown with increased internet use since the COVID-19 lockdown. Farooq et al tracked the effects of social media, news websites and emails as well as the living situation on the individual level, and the intention of self-isolation during the pandemic in a panel of 225 participants ${ }^{108}$ and found that while frequent social media use contributed to information overload and cyberchondria, it increased propensity for self-isolation. McNeill et al similarly studied the effects of tweets and found that social media played a role in the motivation to conform to health measures. ${ }^{109}$ Based on populational surveys, Kowalski et al reported that conspiracy beliefs of COVID-19 were negatively associated with adherence to self-isolation guidelines in Poland. ${ }^{110}$ To motivate members of the public to self-isolate in a healthy way, a combination of lowering perceived response costs and clear information about the severity of risks should be implemented. ${ }^{108111}$ It is also suggested that messages of the severity of disease be disseminated via reliable official networks such as government outreach, news and journalism, while social media focuses on hopeful messages and recommended health measures. $^{112}$

\section{Legal issues}

In Singapore, the Infectious Diseases Act provides a legal basis to prosecute those who are noncompliant, with resulting fines and/or imprisonment. ${ }^{64}$ Similarly, the Communicable Disease Control Act in Taiwan mandates the government to implement effective measures in COVID-19 controls including FTTIS. ${ }^{60}$

In the USA, the Public Health Service Act empowers the Surgeon General (delegated to the CDC) to enforce quarantine and isolation and provide medical care for detained individuals. Court rulings establish that quarantine cannot be imposed in a way that is racially discriminatory, and governments are required to have a strong legal basis for imposed restrictions. ${ }^{78}$

\section{Public cooperation}

The effective control of the highly contagious COVID-19 epidemic relies on collaboration by individuals to voluntarily provide their information and comply with preventive measures. In low or middle-income countries, low public trust due to government corruption impedes public cooperation to observe FTTI regulations. ${ }^{113}$ The potential undesirable outcome of telling the truth, such as adverse immigration consequences for noncitizens, deportation of undocumented migrants or refusal of entry for passengers with a fever, can be a barrier for these individuals, who may be at high risk of infection, to seek care or provide accurate information. ${ }^{78}$ Results from a multinational survey in France, Germany, Italy, the UK and USA showed high public acceptability of a contact-tracing app. ${ }^{114}$ Perceived benefits, self-efficacy ${ }^{115}$ and trust ${ }^{116}$ were positively associated with contact tracing apps uptake. Special protection of personal information is needed, with the goal of facilitating trust, prioritising care and encouraging individuals to cooperate and to prevent further transmission of the disease.

\section{Economic support}

To ease the economic pressure and uncertainty during large-scale quarantine, governments have established different schemes to support individuals and families. Income compensation during quarantine has shown to double the compliance with self-isolation. ${ }^{117}$ East Asian countries have provided support schemes for employers, employees and households. ${ }^{54}$ In the USA, the federal government passed the Families First Coronavirus Response Act, including paid sick leave and unemployment insurance. ${ }^{96}$ Countries implemented funding, financial stimulus, loans, aid packages to support the economy ${ }^{83}$ or individual livelihood. ${ }^{118}$ In the UK, national job retention schemes and financial support schemes 
have been rolled out to support individuals and businesses during the COVID-19 epidemic. ${ }^{96}$

\section{DISCUSSION}

We identified in our systematic review the core elements for an effective FTTIS system necessary to interrupt the spread of a novel infectious disease, as in the COVID-19 pandemic. The optimal strategies for a successful FTTIS during COVID-19 rapid response include:

\section{Finding}

Border controls, restricted entry and inbound traveller quarantine applied early in the pandemic have both reduced the spread of COVID-19 and facilitated case finding. Testing high-risk populations (such as healthcare workers or residents of long-term care facilities) is important for active case finding, especially during the early stages.

\section{Testing}

During the initial response to a pandemic, repurposing of an existing laboratory network for testing should be implemented where possible while establishing new testing sites to meet increasing needs as the pandemic progresses. Pooled testing can be used to improve testing efficiency. Repeated testing may be necessary to minimise false-negative results.

\section{Trace and isolate}

Digital tools such as apps, GPS and mobile geopositioning data have been deployed effectively by some countries for contact tracing and to monitor compliance with selfisolation. As an individual infected by SARS-CoV-2 can actively shed the virus 3 weeks or longer after symptoms onset, the duration of quarantine may vary. A 2-week quarantine period with extension when required has been adopted by various countries.

\section{Support}

Support can be in the form of mental health or physical health promotion or monetary aid, such as timely and adequate information to reduce uncertainty and anxiety, providing adequate supplies or allowance for individuals. These support measures also facilitate public trust that is fundamental in the joint COVID-19 response in the population, as it relies on individuals to voluntarily provide their information and adhere to disease control regulations.

\section{Integrated system}

Although these measures, often combined, are implemented in different countries, the level of preparedness, timely implementation and scale-up of effective measures made the difference between ${ }^{119}$ or within ${ }^{120}$ countries. For incidence, insufficient testing and quarantine led to the undetected wide transmission of SARS-CoV-2, resulting in high case fatality during the first month of COVID-19 epidemic in Italy ${ }^{22}$ and similarly in the Middle East $^{20}$ or during the first wave in the UK. ${ }^{121}$

A successful FTTIS requires seamless integration of all the components and the ability to integrate vulnerable populations or ethnic minorities. Linked health service data can contribute to the cooperation and communications among responsible authorities (central and local government, borders, laboratories and the medical care system) through real-time data sharing and dashboards.

\section{Public collaboration}

In addition to support for individuals, regular press conferences by the central outbreak control team to provide a briefing on the progress of the pandemic, changes in policies and correcting misinformation can increase public awareness of the need for FTTIS. Open and balanced discussions on public concerns, such as personal data privacy and protection and limitations on individuals' freedom for the public good, are essential.

\section{Agile process}

The uncertainty and rapid progression of the pandemic make it important to have the flexibility to adapt rapidly to emerging challenges. A rolling-wave approach to planning can help authorities learn, apply and refine tools for prevention and control, with an agile process allowing better responses to emergencies.

\section{Strengths}

We found no published systematic review addressing simultaneously the finding, test, tracing, isolation and support in the early response of COVID-19. Results of our review thus may inform countries facing future pandemics.

\section{Limitations}

We reviewed studies published in English, thus enable the review team to cross-validate the results. Although information documented in other languages might be missed, our search included studies from all regions of the world. Our review focused on FTTIS in the early phase of the pandemic. However, going forward, it will be important to draw on lessons learnt later in the pandemic.

\section{Conclusion}

This comprehensive systematic review identified effective strategies for a successful FTTIS system to interrupt the spread of a novel infectious disease. These include border controls, restricted entry, inbound traveller quarantine and screening for case finding; repeated testing to minimise false diagnoses and pooled testing in resourcelimited circumstances; extended quarantine period and the use of digital tools for contact tracing and selfisolation. Support for mental or physical health and livelihood is needed for individuals undergoing self-isolation/ quarantine. An integrated system with rolling-wave planning can best use effective FTTIS tools to respond to the fast-changing COVID-19 pandemic. Results of the review 
may inform countries considering implementing these measures.

\section{Author affiliations}

${ }^{1}$ Institute of Health Informatics, University College London, London, UK

${ }^{2}$ Department of Chemical Engineering, University College London, London, UK

${ }^{3}$ Bartlett School of Planning, University College London, London, UK

${ }^{4}$ Charité Universitätsmedizin Berlin, Berlin, Germany

${ }^{5}$ British Institute of International and Comparative Law, London, UK

${ }^{6}$ Department of Medicine, College of Medicine, Fu-Jen Catholic University, New Taipei City, Taiwan

${ }^{7}$ Big Data Research Center, College of Medicine, Fu-Jen Catholic University, New

Taipei City, Taiwan

${ }^{8}$ Diabetes Research Centre, University of Leicester, Leicester, UK

${ }^{9}$ European Centre on Health of Societies in Transition, London School of Hygiene and Tropical Medicine, London, UK

${ }^{10}$ Centre for Behaviour Change, Department of Clinical, Educational and Health Psychology, University College London, London, UK

${ }^{11}$ Division of Infection and Immunity, University College London, London, UK

\section{Twitter Sheng-Chia Chung @ShengChiaChung1 and Martin McKee @martinmckee}

Acknowledgements The study received no funding. The authors thank the list of questions raised by the Independent SAGE team (www.independentsage.org). We also acknowledge discussions within the EPSRC funded i sense consortium (https:// www.i-sense.org.uk/). KK is supported by the National Institute for Health Research (NIHR) Applied Research Collaboration East Midlands (ARC EM) and the NIHR Leicester Biomedical Research Centre (BRC).

Collaborators Sheng-Chia Chung, Sushila Marlow, Nicholas Tobias, Alessio Alogna, Ivano Alogna, San-Lin You, Khunti Kamlesh, Martin Mckee, Susan Michie, Deenan Pillay.

Contributors S-CC, SMa, NT performed the acquisition, analysis and interpretation of data for the work and draft the manuscript. All authors (S-CC, SMa, NT, AA, IA, S-LY, KK, MM, SM, DP) contributed to the conception and design of the work, further data interpretation, revising it critically for important intellectual content, provided final approval of the version to be published and agreement to be accountable for all aspects of the work in ensuring that questions related to the accuracy or integrity of any part of the work are appropriately investigated and resolved. S-CC had final responsibility for the decision to submit for publication.

Funding DP is funded through the EPSRC i sense consortium, and other authors receive no funding for the study.

Competing interests None declared.

Patient consent for publication Not required.

Provenance and peer review Not commissioned; externally peer reviewed.

Data availability statement Data are available in a public, open access repository.

Supplemental material This content has been supplied by the author(s). It has not been vetted by BMJ Publishing Group Limited (BMJ) and may not have been peer-reviewed. Any opinions or recommendations discussed are solely those of the author(s) and are not endorsed by BMJ. BMJ disclaims all liability and responsibility arising from any reliance placed on the content. Where the content includes any translated material, BMJ does not warrant the accuracy and reliability of the translations (including but not limited to local regulations, clinical guidelines, terminology, drug names and drug dosages), and is not responsible for any error and/or omissions arising from translation and adaptation or otherwise.

Open access This is an open access article distributed in accordance with the Creative Commons Attribution Non Commercial (CC BY-NC 4.0) license, which permits others to distribute, remix, adapt, build upon this work non-commercially, and license their derivative works on different terms, provided the original work is properly cited, appropriate credit is given, any changes made indicated, and the use is non-commercial. See: http://creativecommons.org/licenses/by-nc/4.0/.

\section{ORCID iDs}

Sheng-Chia Chung http://orcid.org/0000-0001-6025-9207

Martin McKee http://orcid.org/0000-0002-0121-9683
REFERENCES

1 Royal Society DELVE Initiative. Test, trace, isolate. Available: https:// rs-delve.github.io/reports/2020/05/27/test-trace-isolate.html

2 Khunti K, Pollock AM, Pareek M. Find, test, trace, isolate and support programmes need to be localised and culturally tailored to reach ethnic minority populations. Available: https://blogs.bmj.com/ bmj/2020/07/21/find-test-trace-isolate-and-support-programmesneed-to-be-localised-and-culturally-tailored-to-reach-ethnicminority-populations/

3 New and Emerging Respiratory Virus Threats Advisory Group. Key issues for contact tracing for consideration by NERVTAG. Available: https://assets.publishing.service.gov.uk/government/uploads/ system/uploads/attachment_data/file/890187/s0275-key-issuesfor-contact-tracing-for-consideration-by-nervtag-230420-sage30. pdf [Accessed 23 Apr 2020].

4 Kendall MML, Abeler-Dorner L, Wymant C. COVID-19 incidence and $R$ decreased on the Isle of Wight after the launch of the test, trace, isolate programme. Medrxiv.

5 Thorneloe RET, Fynn W, Daly M. Scoping review of mobile phone APP uptake and engagement to inform digital contact tracing tools for COVID-19. Psyarxiv.

6 Vize R. How the erosion of our public health system hobbled England's covid-19 response. BMJ 2020;369:m1934.

7 Ebrahim SH, Zhuo J, Gozzer E, et al. All hands on Deck: a synchronized whole-of-world approach for COVID-19 mitigation. Int $J$ Infect Dis 2020;98:208-15.

8 The British Pshycological Society. Briefing - Behavioural science and success of the proposed UK digital contact tracing application for Covid-19. Available: https://www.bps.org.uk/sites/www.bps. org.uk/files/Policy/Policy\%20-\%20Files/Behavioural\%20science\% 20-\%20digital\%20contact\%20tracing\%20for\%20Covid-19.pdf

9 Scientific Pandemic Influenza Group on Behaviours. Symptombased contact tracing is likely to reduce adherence to advice to quarantine in comparison to test-based approaches. Available: https://assets.publishing.service.gov.uk/government/uploads/ system/uploads/attachment_data/file/888750/7b._20.04.27_ SPI-B_behavioural_science_notes_on_symptom_vs_test_based approaches_S0260.pdf

10 European Observatory on Health Systems and Policies. COVID response monitor: European observatory on health systems and policies, 2020. Available: https://analysis.covid19healthsystem.org/ [Accessed 14 Jun 2020].

11 Methley AM, Campbell S, Chew-Graham C, et al. PICO, PICOS and SPIDER: a comparison study of specificity and sensitivity in three search tools for qualitative systematic reviews. BMC Health Serv Res 2014;14:579.

12 Noyes J, Booth A, Moore G, et al. Synthesising quantitative and qualitative evidence to inform guidelines on complex interventions: clarifying the purposes, designs and outlining some methods. BMJ Glob Health 2019;4:e000893.

13 Critical Appraisal Skills Programme. CASP qualitative studies checklist [online], 2018. Available: https://casp-uk.net/wp-content/ uploads/2018/01/CASP-Qualitative-Checklist-2018.pdf

14 Nussbaumer-Streit B, Mayr V, Dobrescu Al, et al. Quarantine alone or in combination with other public health measures to control COVID-19: a rapid review. Cochrane Database Syst Rev 2020;4:CD013574.

15 Liu Y, Eggo RM, Kucharski AJ. Secondary attack rate and superspreading events for SARS-CoV-2. Lancet 2020;395:e47.

16 Grassly NC, Pons-Salort M, Parker EPK, et al. Comparison of molecular testing strategies for COVID-19 control: a mathematical modelling study. Lancet Infect Dis 2020;20:1381-9.

17 Aleta A, Martín-Corral D, Pastore Y, et al. Modelling the impact of testing, contact tracing and household quarantine on second waves of COVID-19. Nat Hum Behav 2020;4:964-71.

18 Sjödin H, Wilder-Smith A, Osman S, et al. Only strict quarantine measures can curb the coronavirus disease (COVID-19) outbreak in Italy, 2020. Euro Surveill 2020;25.

19 Omori R, Mizumoto K, Chowell G. Changes in testing rates could mask the novel coronavirus disease (COVID-19) growth rate. Int $J$ Infect Dis 2020;94:116-8.

20 Salameh P. COVID-19 in the eastern Mediterranean region: testing frequency, cumulative cases and mortality analysis. East Mediterr Health $J$ 2020;26:1005-10.

21 Manchein C, Brugnago EL, da Silva RM, et al. Strong correlations between power-law growth of COVID-19 in four continents and the inefficiency of soft quarantine strategies. Chaos 2020;30:041102.

22 Vicentini C, Bazzolo S, Gamba D, et al. Analysis of the fatality rate in relation to testing capacity during the first 50 days of the COVID-19 epidemic in Italy. Am J Trop Med Hyg 2020;103:2382-90. 
23 Edjoc R, Atchessi N, Lien A, et al. Assessing the progression of the COVID-19 pandemic in Canada using testing data and time-dependent reproduction numbers. Can J Public Health 2020;111:926-38.

24 Chen C-M, Jyan H-W, Chien S-C, et al. Containing COVID-19 among 627,386 persons in contact with the diamond Princess cruise SHIP passengers who Disembarked in Taiwan: big data analytics. J Med Internet Res 2020;22:e19540.

25 Hill K, Campbell R, Mutch C, et al. Drive-through testing in COVID-19: experience from NHS Lothian. Clin Med 2020;20:290-1.

26 Lagier JC, Colson P, Tissot Dupont H, et al. Testing the repatriated for SARS-Cov2: should laboratory-based quarantine replace traditional quarantine? Travel Med Infect Dis 2020;34:101624.

27 Smyrlaki I, Ekman M, Lentini A, et al. Massive and rapid COVID-19 testing is feasible by extraction-free SARS-CoV-2 RT-PCR. Nat Commun 2020;11:4812.

28 Bradshaw WJ, Alley EC, Huggins JH, et al. Bidirectional contact tracing could dramatically improve COVID-19 control. Nat Commun 2021;12:232.

29 Binnicker MJ. Emergence of a novel coronavirus disease (COVID-19) and the importance of diagnostic testing: why partnership between clinical laboratories, public health agencies, and industry is essential to control the outbreak. Clin Chem 2020;66:664-6.

30 Gupta N, Potdar V, Praharaj I. Laboratory preparedness for SARSCoV-2 testing in India: Harnessing a network of Virus Research \& Diagnostic Laboratories. Indian J Med Res 2020;151:216-25.

31 Abera A, Belay H, Zewude A, et al. Establishment of COVID-19 testing laboratory in resource-limited settings: challenges and prospects reported from Ethiopia. Glob Health Action 2020;13:1841963.

32 Tolia VM, Chan TC, Castillo EM. Preliminary results of initial testing for coronavirus (COVID-19) in the emergency department. West $J$ Emerg Med 2020;21:503-6.

33 Koh D, Cunningham AC. Counting coronavirus disease 2019 (COVID-19) cases: case definitions, screened populations and testing techniques matter. Ann Acad Med Singapore 2020;49:161-5.

34 Yan Y, Chang L, Wang L. Laboratory testing of SARS-CoV, MERSCoV, and SARS-CoV-2 (2019-nCoV): current status, challenges, and countermeasures. Rev Med Virol 2020;30:e2106.

35 de Wolff T, Pflüger D, Rehme M, et al. Evaluation of pool-based testing approaches to enable population-wide screening for COVID-19. PLoS One 2020;15:e0243692.

36 Deckert A, Bärnighausen T, Kyei NNA. Simulation of pooled-sample analysis strategies for COVID-19 mass testing. Bull World Health Organ 2020;98:590-8.

37 Smalley DL, Cisarik PM, Grantham J, et al. Impact of pool testing in detection of asymptomatic patients with COVID-19. Lab Med 2021;52:e15-16.

38 Abdalhamid B, Bilder CR, McCutchen EL, et al. Assessment of specimen pooling to conserve SARS CoV-2 testing resources. Am J Clin Pathol 2020;153:715-8.

39 Eberhardt JN, Breuckmann NP, Eberhardt CS. Multi-Stage group testing improves efficiency of large-scale COVID-19 screening. $J$ Clin Virol 2020;128:104382.

40 McCombs A, Kadelka C. A model-based evaluation of the efficacy of COVID-19 social distancing, testing and hospital triage policies. PLoS Comput Biol 2020;16:e1008388.

41 Müller M, Derlet PM, Mudry C, et al. Testing of asymptomatic individuals for fast feedback-control of COVID-19 pandemic. Phys Biol 2020;17:065007.

42 Gray N, Calleja D, Wimbush A, et al. Is "no test is better than a bad test"? Impact of diagnostic uncertainty in mass testing on the spread of COVID-19. PLoS One 2020;15:e0240775.

43 Bai AD, Li XX, Alsalem M, et al. Utility of asymptomatic inpatient testing for COVID-19 in a low-prevalence setting: a multicenter point-prevalence study. Infect. Control Hosp. Epidemiol. 2020;41:1233-5.

44 Wei C, Lee CC, Hsu TC, et al. Correlation of population mortality of COVID-19 and testing coverage: a comparison among 36 OECD countries. Epidemiol Infect 2021;149:e1.

45 Mettler SK, Kim J, Maathuis MH. Diagnostic serial interval as a novel indicator for contact tracing effectiveness exemplified with the SARS-CoV-2/COVID-19 outbreak in South Korea. Int J Infect Dis 2020;99:346-51.

46 Malheiro R, Figueiredo AL, Magalhães JP, et al. Effectiveness of contact tracing and quarantine on reducing COVID-19 transmission: a retrospective cohort study. Public Health 2020;189:54-9.
47 Sawano T, Kotera Y, Ozaki A, et al. Underestimation of COVID-19 cases in Japan: an analysis of RT-PCR testing for COVID-19 among 47 prefectures in Japan. QJM 2020;113:551-5.

48 Younes N, Al-Sadeq DW, AL-Jighefee $\mathrm{H}$, et al. Challenges in laboratory diagnosis of the novel coronavirus SARS-CoV-2. Viruses2020;12:582.

49 Zitek T. The appropriate use of testing for COVID-19. West J Emerg Med 2020;21:470-2.

$50 \mathrm{Li} \mathrm{Y,} \mathrm{Yao} \mathrm{L,} \mathrm{Li} \mathrm{J,} \mathrm{et} \mathrm{al.} \mathrm{Stability} \mathrm{issues} \mathrm{of} \mathrm{RT-PCR} \mathrm{testing} \mathrm{of} \mathrm{SARS-}$ CoV-2 for hospitalized patients clinically diagnosed with COVID-19. J Med Virol 2020;92:903-8.

51 Döhla M, Boesecke C, Schulte B, et al. Rapid point-of-care testing for SARS-CoV-2 in a community screening setting shows low sensitivity. Public Health 2020;182:170-2.

52 Gopaul R, Davis J, Gangai L, et al. Practical diagnostic accuracy of nasopharyngeal swab testing for novel coronavirus disease 2019 (COVID-19). West J Emerg Med 2020;21:1-4.

53 Falaschi Z, Danna PSC, Arioli R, et al. Chest CT accuracy in diagnosing COVID-19 during the peak of the Italian epidemic: a retrospective correlation with RT-PCR testing and analysis of discordant cases. Eur J Radiol 2020;130:109192.

54 Aslam H, Rohan H. Fighting COVID-19: lessons from China, South Korea and Japan. Sustainable Development Policy Institute, 2020.

55 Brendish NJ, Poole S, Naidu VV, et al. Clinical impact of molecular point-of-care testing for suspected COVID-19 in hospital (COV19POC): a prospective, interventional, non-randomised, controlled study. Lancet Respir Med 2020;8:1192-200.

56 Indenbaum V, Koren R, Katz-Likvornik S, et al. Testing IgG antibodies against the RBD of SARS-CoV-2 is sufficient and necessary for COVID-19 diagnosis. PLoS One 2020;15:e0241164.

57 Brooks ZC, Das S. COVID-19 testing. Am J Clin Pathol 2020;154:575-84

58 Jung J, Jang H, Kim HK, et al. The importance of mandatory COVID-19 diagnostic testing prior to release from quarantine. J Korean Med Sci 2020;35:e314.

59 Dinnes J, Deeks JJ, Berhane S, et al. Rapid, point-of-care antigen and molecular-based tests for diagnosis of SARS-CoV-2 infection. Cochrane Database Syst Rev 2021;3:Cd013705.

60 Han E, Chiou S-T, McKee M, et al. The resilience of Taiwan's health system to address the COVID-19 pandemic. EClinicalMedicine 2020;24:100437.

61 National Health Insurance Administration. Enhanced patient safety and information security both through the NHI MediCloud system. Available: https://www.nhi.gov.tw/english/News_Content. asp $\mathrm{n} n=996 \mathrm{D} 1 \mathrm{~B} 4 \mathrm{~B} 5 \mathrm{DC} 48343 \& \mathrm{sms}=\mathrm{F} 0 \mathrm{EAFEB} 716 \mathrm{DE} 7 \mathrm{FFA} \& \mathrm{~s}=$ 148EAC979AAE3F75

62 National Health Insurance Administration. Information on departures/transits to high-risk areas is now included in the travel history notification list, thus effectively closing gaps in disease prevention. Available: https://www.nhi.gov.tw/ English/News_Content.aspx?n=996D1B4B5DC48343\&sms= F0EAFEB716DE7FFA\&s=00AF96BA7D327FF5

63 Canova V, Lederer Schläpfer $\mathrm{H}$, Piso RJ, et al. Transmission risk of SARS-CoV-2 to healthcare workers -observational results of a primary care hospital contact tracing. Swiss Med Wkly 2020;150:w20257.

64 Chiew CJ, Li Z, Lee VJ. Reducing onward spread of COVID-19 from imported cases: quarantine and 'stay at home' measures for travellers and returning residents to Singapore. J Travel Med 2020;27.

65 Ferretti L, Wymant C, Kendall M, et al. Quantifying SARS-CoV-2 transmission suggests epidemic control with digital contact tracing Science2020;368:eabb6936.

66 Bilinski A, Mostashari F, Salomon JA. Modeling contact tracing strategies for COVID-19 in the context of relaxed physical distancing measures. JAMA Netw Open 2020;3:e2019217.

67 Kretzschmar ME, Rozhnova G, Bootsma MCJ, et al. Impact of delays on effectiveness of contact tracing strategies for COVID-19: a modelling study. Lancet Public Health 2020;5:e452-9.

68 Eilersen A, Sneppen K. Cost-benefit of limited isolation and testing in COVID-19 mitigation. Sci Rep 2020;10:18543.

69 Wells CR, Townsend JP, Pandey A, et al. Optimal COVID-19 quarantine and testing strategies. Nat Commun 2021;12:356.

70 Park Y, Huh IS, Lee J, et al. Application of Testing-TracingTreatment strategy in response to the COVID-19 outbreak in Seoul, Korea. J Korean Med Sci 2020;35:e396.

71 Keeling MJ, Hollingsworth TD, Read JM. Efficacy of contact tracing for the containment of the 2019 novel coronavirus (COVID-19). J Epidemiol Community Health 2020;74:861-6. 
72 Park S, Choi GJ, Ko H. Information technology-based tracing strategy in response to COVID-19 in South Korea-Privacy controversies. JAMA 2020;323:2129.

73 Klonowska, Klaudia, and Pieter Bindt. The COVID-19 pandemic: two waves of technological responses in the European Union. Hague centre for strategic studies 2020

74 Klimburg A, Faesen L, Verhagen P. Pandemic mitigation in the digital age: digital epidemiological measures to combat the coronavirus pandemic. Hague Centre for Strategic Studies 2020.

75 Szocska M, Pollner P, Schiszler I, et al. Countrywide population movement monitoring using mobile devices generated (big) data during the COVID-19 crisis. Sci Rep 2021;11:5943.

76 Wang S, Ding S, Xiong L. A new system for surveillance and digital contact tracing for COVID-19: spatiotemporal reporting over network and GPs. JMIR Mhealth Whealth 2020;8:e19457.

77 Webster RK, Brooks SK, Smith LE, et al. How to improve adherence with quarantine: rapid review of the evidence. Public Health 2020;182:163-9.

78 Parmet WE, Sinha MS. Covid-19 - the law and limits of quarantine. N Engl J Med 2020;382:e28.

79 Lei L, Huang X, Zhang S, et al. Comparison of prevalence and associated factors of anxiety and depression among people affected by versus people unaffected by quarantine during the COVID-19 epidemic in southwestern China. Med Sci Monit 2020;26:e924609

80 Razai MS, Oakeshott P, Kankam H, et al. Mitigating the psychological effects of social isolation during the covid-19 pandemic. BMJ2020;369:m1904.

81 Peçanha T, Goessler KF, Roschel H, et al. Social isolation during the COVID-19 pandemic can increase physical inactivity and the globa burden of cardiovascular disease. Am J Physiol Heart Circ Physiol 2020;318:H1441-6.

82 Reiss S, Franchina V, Jutzi C, et al. From anxiety to actionExperience of threat, emotional states, reactance, and action preferences in the early days of COVID-19 self-isolation in Germany and Austria. PLoS One 2020;15:e0243193.

83 Desson Z, Lambertz L, Peters JW, et al. Europe's Covid-19 outliers: German, Austrian and Swiss policy responses during the early stages of the 2020 pandemic. Health Policy Technol 2020;9:405-18.

84 Banskota S, Healy M, Goldberg EM. 15 smartphone Apps for older adults to use while in isolation during the COVID-19 pandemic. West J Emerg Med 2020;21:514-25.

85 Meinert E, Milne-Ives M, Surodina S, et al. Agile requirements engineering and software planning for a digital health platform to engage the effects of isolation caused by social distancing: case study. JMIR Public Health Surveill 2020;6:e19297.

86 Yasaka TM, Lehrich BM, Sahyouni R. Peer-to-Peer contact tracing: development of a Privacy-Preserving smartphone APP. JMIR Mhealth Uhealth 2020;8:e18936.

87 Ekong I, Chukwu E, Chukwu M. COVID-19 mobile positioning data contact tracing and patient privacy regulations: exploratory search of global response strategies and the use of digital tools in Nigeria. JMIR Mhealth Uhealth 2020;8:e19139.

88 Lai S, Farnham A, Ruktanonchai NW, et al. Measuring mobility, disease connectivity and individual risk: a review of using mobile phone data and mHealth for travel medicine. J Travel Med 2019;26.

89 JW X, Wang XY, Qin Z. Deep thought of COVID-19 based on diamond princess's quarantine and home quarantine. Eur Rev Med Pharmacol Sci 2020;24:4027-9.

90 Kakimoto K, Kamiya H, Yamagishi T, et al. Initial investigation of transmission of COVID-19 among Crew members during quarantine of a cruise SHIP - Yokohama, Japan, February 2020. MMWR Morb Mortal Wkly Rep 2020;69:312-3.

91 Yamahata Y, Shibata A. Preparation for quarantine on the cruise SHIP diamond Princess in Japan due to COVID-19. JMIR Public Health Surveill 2020;6:e18821.

92 Wang J, Feng H, Zhang S, et al. SARS-CoV-2 RNA detection of hospital isolation wards hygiene monitoring during the coronavirus disease 2019 outbreak in a Chinese Hospital. Int J Infect Dis 2020;94:103-6.

93 Keeley AJ, Evans C, Colton H, et al. Roll-Out of SARS-CoV-2 testing for healthcare workers at a large NHS Foundation trust in the United Kingdom, March 2020. Euro Surveill 2020;25.

94 Cheng $\mathrm{H}-\mathrm{Y}$, Jian S-W, Liu D-P, et al. Contact tracing assessment of COVID-19 transmission dynamics in Taiwan and risk at different exposure periods before and after symptom onset. JAMA Intern Med 2020;180:1156-63.

95 Yen M-Y, Schwartz J, Chen S-Y, et al. Interrupting COVID-19 transmission by implementing enhanced traffic control bundling: implications for global prevention and control efforts. J Microbiol Immunol Infect 2020;53:377-80.
96 Yen M-Y, Lin Y-E, Lee C-H, et al. Taiwan's traffic control bundle and the elimination of nosocomial severe acute respiratory syndrome among healthcare workers. J Hosp Infect 2011;77:332-7.

97 Huang Z, Guo H, Lee Y-M, et al. Performance of digital contact tracing tools for COVID-19 response in Singapore: cross-sectional study. JMIR Mhealth Uhealth 2020;8:e23148.

98 Jian S-W, Cheng $\mathrm{H}-\mathrm{Y}$, Huang $\mathrm{X}-\mathrm{T}$, et al. Contact tracing with digital assistance in Taiwan's COVID-19 outbreak response. Int Journal of Dis 2020;101:348-52.

99 Ruebush E, Fraser MR, Poulin A, et al. COVID-19 case investigation and contact tracing: early lessons learned and future opportunities. $J$ Public Health Manag Pract 2021;27:S87-97.

100 Rahaman KR, Mahmud MS, Mallick B. Challenges of testing COVID-19 cases in Bangladesh. Int $J$ Environ Res Public Health 2020;17:6439.

101 Wu DC, Jha P, Lam T, et al. Predictors of self-reported symptoms and testing for COVID-19 in Canada using a nationally representative survey. PLoS One 2020;15:e0240778.

102 Mark K, Steel K, Stevenson J, et al. Coronavirus disease (COVID-19) community testing team in Scotland: a 14-day review, 6 to 20 February 2020. Euro Surveill 2020;25.

103 Office for National Statistics. Internet users, UK, 2019. Available: https://www.ons.gov.uk/businessindus- tryandtrade/ itandinternetindustry/bulletins/internetusers/2019

104 Jonker M, de Bekker-Grob E, Veldwijk J, et al. COVID-19 contact tracing Apps: predicted uptake in the Netherlands based on a discrete choice experiment. JMIR Mhealth Uhealth 2020;8:e20741.

105 Zhang M-X, Yan H-F, Wu J-Y, et al. Quarantine vehicle scheduling for transferring high-risk individuals in epidemic areas. Int J Environ Res Public Health 2020;17:2275.

106 Chanda A. COVID-19 in India: transmission dynamics, epidemiological characteristics, testing, recovery and effect of weather. Epidemiol Infect 2020;148:e182.

107 Li S, Feng B, Liao W, et al. Internet use, risk awareness, and demographic characteristics associated with engagement in preventive behaviors and testing: cross-sectional survey on COVID-19 in the United States. J Med Internet Res 2020;22:e19782.

108 Farooq A, Laato S, Islam AKMN. Impact of online information on Self-Isolation intention during the COVID-19 pandemic: crosssectional study. J Med Internet Res 2020;22:e19128.

109 McNeill A, Harris PR, Briggs P. Twitter influence on UK vaccination and antiviral uptake during the $2009 \mathrm{H} 1 \mathrm{~N} 1$ pandemic. Front Public Health 2016;4:26.

110 Kowalski J, Marchlewska M, Molenda Z, et al. Adherence to safety and self-isolation guidelines, conspiracy and paranoia-like beliefs during COVID-19 pandemic in Poland - associations and moderators. Psychiatry Res 2020;294:113540.

111 Xie K, Liang B, Dulebenets MA, et al. The impact of risk perception on social distancing during the COVID-19 pandemic in China. Int $J$ Environ Res Public Health 2020;17:6256.

112 Schrager J, Schuler K, Wright D, et al. Development and usability testing of a web-based COVID-19 Self-triage platform. West J Emerg Med 2020;21:1054-8.

113 Ezeibe CC, Ilo C, Ezeibe EN, et al. Political distrust and the spread of COVID-19 in Nigeria. Glob Public Health 2020;15:1753-66.

114 Altmann S, Milsom L, Zillessen H, et al. Acceptability of App-Based contact tracing for COVID-19: Cross-Country survey study. JMIR Mhealth Uhealth 2020;8:e19857.

115 Walrave M, Waeterloos C, Ponnet K. Adoption of a contact tracing APP for containing COVID-19: a health belief model approach. JMIR Public Health Surveill 2020;6:e20572.

116 Kaspar K. Motivations for social distancing and APP use as complementary measures to combat the COVID-19 pandemic: quantitative survey study. J Med Internet Res 2020;22:e21613.

117 Bodas M, Peleg K. Self-Isolation compliance in the COVID-19 era influenced by compensation: findings from a recent survey in Israel. Health Aff 2020;39:936-41.

118 Bodas M, Peleg K. Income assurances are a crucial factor in determining public compliance with self-isolation regulations during the COVID-19 outbreak - cohort study in Israel. Isr J Health Policy Res 2020;9:54.

119 DB S, Undi M, Annadani R, et al. Comparison of measures adopted to combat COVID 19 pandemic by different countries in who regions. Indian J Community Health 2020;32:288-99.

120 Chiu WA, Fischer R, Ndeffo-Mbah ML. State-level needs for social distancing and contact tracing to contain COVID-19 in the United States. Nat Hum Behav 2020:4:1080-90.

121 Frowde R, Dove ES, Laurie GT. Fail to prepare and you prepare to fail: the human rights consequences of the UK government's inaction during the COVID-19 pandemic. Asian Bioeth Rev 2020:1-22. 